\title{
Towards the development of a standard test procedure for mouthguard assessment
}

\author{
A Greasley, B Karet
}

\begin{abstract}
A simulated upper jaw, made from a rubber arch containing replaceable ceramic teeth and a renewable composite jawbone, offers promise in assessing the performance of custom made mouthguard designs. Impact tests, involving precise assessment of jaw and tooth fractures caused by projectiles of various energies and profiles, simulate conditions that approximate to common clinical observation. Such conditions offer the most sensitive indices for assessing both improved mouthguard designs and product quality and reliability. Damage caused by the dissipation of the impact energy may be transferred within this simulated oral cavity by minor changes to the impact conditions.

(BrF Sports Med 1997;31:31-35)
\end{abstract}

Keywords: mouthguard; simulated jaw; impact testing.

Many have reported on the relative advantages of custom made mouthguards worn during participation in contact sports. ${ }^{1-5}$ Such reports are usually based on clinical assessment of injuries sustained. The production methods used to manufacture these guards have also received attention. ${ }^{6}$ However, there are no standards governing the production quality and effectiveness of such custom made guards. A recent study attempted to predict the best type of material and wall thicknesses of mouthguards from ball impact forces generated in a rigid load cell cushioned by various thicknesses of test material. ${ }^{7}$ The impact forces generated in such situations are primarily determined by the rate of change of momentum in the impacting body. Not surprisingly thicker cushioning layers gave lower impact forces. The conversion of this force into a stress distribution is of much more clinical interest and to achieve this, carbon paper was included in the assembly to measure the contact areas involved. It is not clear from the work why it was recommended that an insert of more rigid material should be placed within the occlusion surface of the guard. The authors also express great concern regarding the thinning that occurs when custom made mouthguards and "boil and bite" guards are shaped. This is most pronounced on the occlusion surface. Hence they claim that this manufacturing feature results in much higher occlusive forces during an impact. However, in practice the generating forces are rarely applied directly to the occlusion surface. Other workers have mounted strain gauges on mouthguards and monitored responses during in vitro testing. ${ }^{8}$ In the build up to the work presented here, photoelasticity and brittle lacquers were used to investigate stresses generated within mouthguards mounted on simulated jaws during impact. However, using currently available methods the results were inconclusive.

Clinicians promote the use of two or double mouthguards to protect both the upper and lower jaw. This helps to minimise soft tissue damage and the risk of concussion, but mouthguards of this type have proved almost universally unacceptable because they introduce breathing and communication difficulties, especially in team sports (Simmons A, personal communication). Hence clinicians have generally resorted to providing single mouthguards fitted to the upper teeth. Because of the lack of in depth study, mouthguard manufacturers and clinicians follow practices that have been accumulated by experience. A single mouthguard constructed using current practices, which are extracts from the review by Scott $e t$ $a l,{ }^{1}$ is described as follows:

(1) It should enclose the maxillary teeth to the distal surface of the second molars.

(2) Thickness should be $3 \mathrm{~mm}$ on the labial aspects, $2 \mathrm{~mm}$ on the occlusal aspect, and $1 \mathrm{~mm}$ on the palatal aspect.

(3) The labial flange should extend to within 2 $\mathrm{mm}$ of the vestibular reflection.

(4) The palatal flange should extend about 10 $\mathrm{mm}$ above the gingival margin.

(5) The edge of the labial flange should be rounded in cross section whereas the palatal edge is tapered.

In order to gain direct measurements of the performance characteristics of such guards, impact tests have been conducted on custom made mouthguards, manufactured in close, but not exact, agreement with the above "best practice". They are constructed directly onto a "standardised jaw". In the long term it may be possible for regulating authorities to supply such a "standard jaw" to potential manufacturers of mouthguards and have the subsequent jaw and custom fitted mouthguard impact tested under controlled conditions in order to assess the effectiveness of the design, the materials used, and the manufacturing method. Such tests would take all material properties, design details, and manufacturing quality into consideration. This would provide a more 


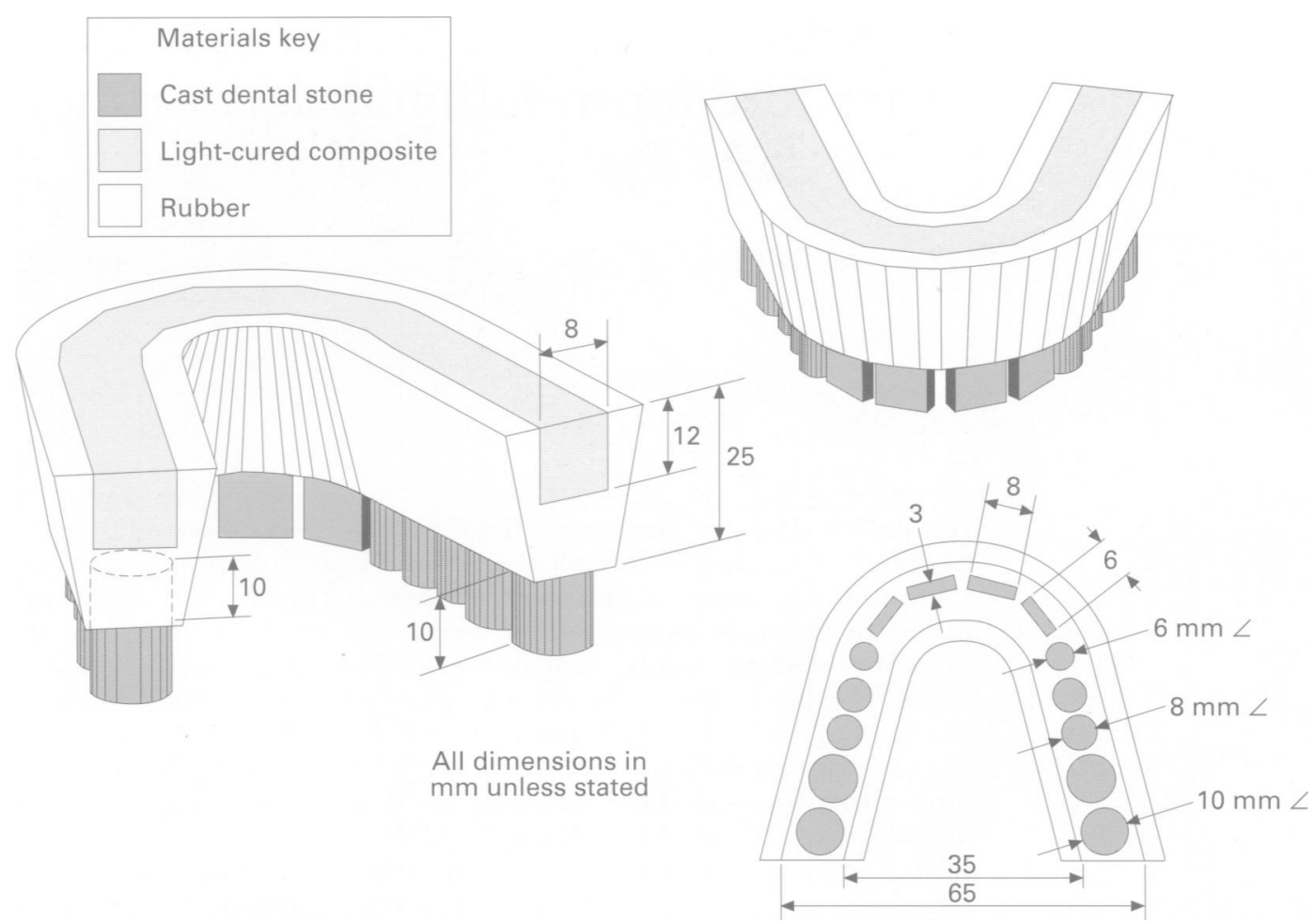

Figure 1 Standard jaw configuration.

rational approach to mouthguard development to be used alongside injury analysis.

\section{Methods}

The standard jaw is based upon an arch of rubber (Shore Hardness 94) containing a rebate to accept a simulated jawbone and blind ended sockets into which simulated and idealised teeth shapes are inserted. The jawbone is cast or cured in situ, depending upon the material used. The idealised teeth are cast or cured in separate moulds. The finished teeth are a snug push fit into the rubber jaw sockets.

The novel overall idealisation and dimensions are as shown in fig 1 . Teeth were cast in separate moulds using a dental stone of fracture toughness $\mathrm{K}_{\mathrm{IC}}=0.5(\mathrm{SD} 0.1) \mathrm{MN}$ $\mathrm{m}^{-3 / 2}$. The jawbone was either cast in situ using the same dental stone or built up using $2 \mathrm{~mm}$ layers of a light cured posterior composite $\left[\mathrm{K}_{\mathrm{IC}}\right.$ $\left.=3.0(0.2) \mathrm{MN} \mathrm{m}^{-3 / 2}\right]$. These fracture tough-

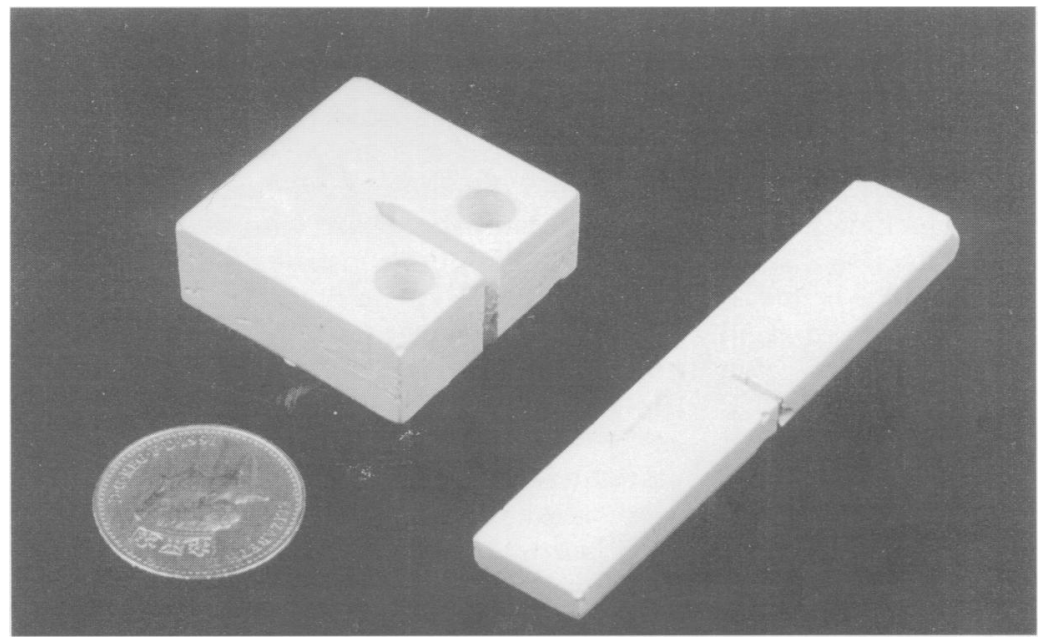

Figure 2 Fracture toughness specimens for standard jaw ceramic components. ness estimates were made using both compact tension and three point bend, single edge notch specimens, as shown in fig 2 , conducted on material prepared under identical conditions. The fracture toughness values encompass the range of values observed in vivo. Calcium hydroxyapatite has a $\mathrm{K}_{\mathrm{IC}}$ in the range $0.2-1.0$ $\mathrm{MN} \mathrm{m} \mathrm{m}^{-3 / 2}$ depending upon the porosity level. ${ }^{9}$ Dentin and enamel have toughness of $4.3 \mathrm{MN}$ $\mathrm{m}^{-3 / 2}$ and $2.7 \mathrm{MN} \mathrm{m}^{-3 / 2}$ respectively. ${ }^{10}$ The dimensions of the teeth and particularly the jawbone are idealised and simplified. Comparisons of how the rubber mounting method compares to the physiological performance of teeth in jaw sockets is open to criticism.

Once complete, standard jaws were fitted with transparent custom made ethyl vinyl acetate (EVA) mouthguards following the recommendations given earlier, with the exception that the guards were trimmed to finish at the centre of the first molar instead of the second molar. The particular manufacturers used feel that the additional protection offered by extending the guard further backwards adds negligible impact performance but reduces breathing, palatal cooling, and speech. The finished guarded jaw is shown in fig 3 .

It was necessary to use two standard jaws and four identical mouthguards in this study. However, the results did not appear to be significantly influenced by the minor manufacturing variations within these assemblies. The jaw and mouthguard assembly was then mounted in a spring loaded device and subjected to impact from free falling hardwood projectiles, weighted to give certain kinetic energies on impact (impact velocity $=6.25$ $\left.\mathrm{ms}^{-1}, \mathrm{E}=1 / 2 \mathrm{mv}^{2}\right)$. The projectiles also had the following end profiles chosen to simulate the types of impact encountered by mouthguard users: 


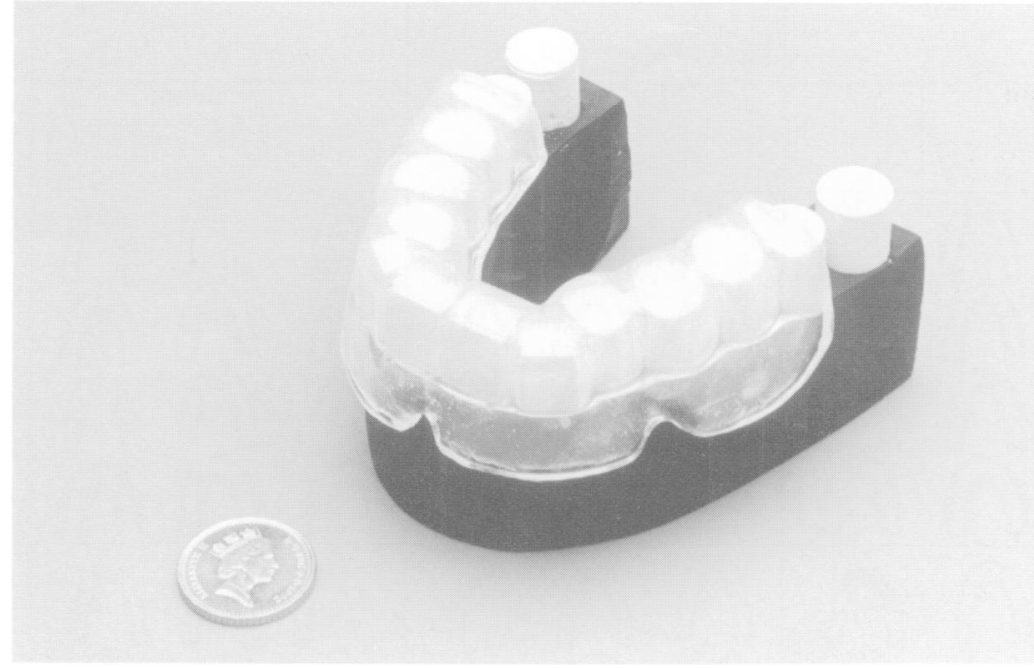

Figure 3 Standard jaw fitted with custom made transparent EVA mouthguard.

(1) Flat ended: boxing, impacts with walls, ground and floors, etc.

(2) Hemispherical (40 $\mathrm{mm}$ radius): cricket ball, hockey ball or puck, headbutts, etc.

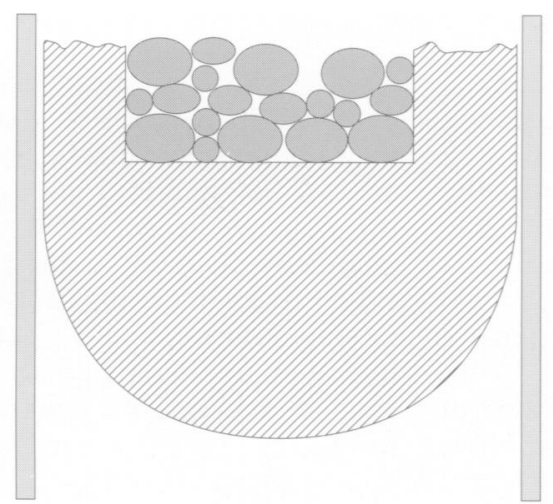

Weighted projectile in guide

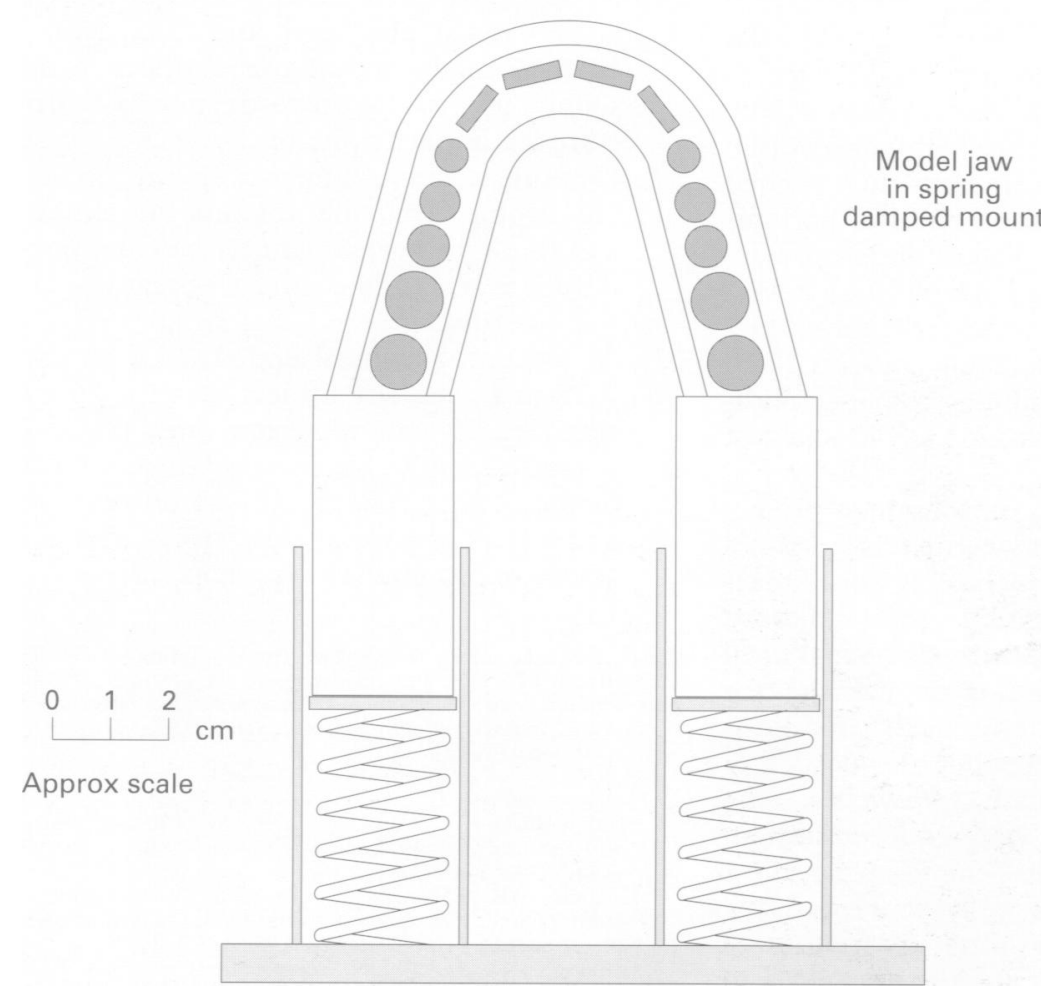

Figure 4 Standard jaw mounted for impact trials.
(3) Rounded cone with a $5 \mathrm{~mm}$ tip radius: hockey stick, squash racket, cricket bat, elbows, etc.

The two compression spring mounts had a combined spring constant of $1 \mathrm{~kg} \mathrm{~mm}^{-1}$ up to the maximum deflection of $16 \mathrm{~mm}$. A simple mechanics analysis revealed that the rate of decrease of momentum using such springs creates forces of $1.3 \mathrm{kN}, 0.98 \mathrm{kN}$, and $0.65 \mathrm{kN}$ when the impact energies are $20 \mathrm{~J}, 15 \mathrm{~J}$, and 10 $\mathrm{J}$ respectively.

The overall layout is shown in fig 4 . All tests were conducted in a heated chamber at $37^{\circ} \mathrm{C}$ in order to put the mouthguard material into its relevant thermoelastic state. For the round ended projectiles the initial point of impact was at the labial-gingival shoulder between the central incisors.

The teeth and jawbone were inspected for fractures after each test and replaced if any cracks or fractures were found. Control samples were also tested under identical conditions but without any mouthguard.

\section{Results and discussion}

Figure 5 shows the average results of three tests under each condition. Broken teeth are blacked out from their relevant position in the schematic arch and fractures across the simulated jawbone are shown where they have occurred. The presence of these fractures is used to estimate the amount of impact energy transmitted to the teeth and jaw during impact. The way this complex elastic-viscoelastic assembly converts the impact force depends and how quickly the momentum of the projectile is dissipated (force $=$ rate of change of momentum) and how this external force is reacted by the internal stress distribution. Careful observation of the data reveals that the standard jaw with the tougher jawbone $\left(\mathrm{K}_{\mathrm{IC}}=3 \mathrm{MN} \mathrm{m}^{-3 / 2}\right)$ is more representative of clinical observations, where fractured teeth are usually observed without fractures occurring to the jawbone. This is seen in both the guarded and unguarded condition. Tests on the simulated jaws with the lower fracture toughness jawbone revealed that at the lowest impact energy it was possible to fracture the jawbone without fracturing teeth, even in the unguarded condition (top left of fig 5). This is contrary to common clinical observation and may be due to the idealised impact direction coupled with the size of the labial-gingival shoulder in the rubber arch. This protrusion may be more robust than in the oral situation and helps in creating bending stresses rather than compressing and transmitting the blow to the teeth.

A subsidiary and unexpected observation was that rounded projectiles often caused the mouthguards to detach in the latter stages of the impact. This could not be attributed to the poor fit of the guards since they appeared to be as tight a fit as is observed in vivo. Lateral movement of the spring loaded base, which occurs during the tests, may contribute to this phenomenon. However, it occurred so late during the impact that it did not influence the damage imparted to the standard jaw. 


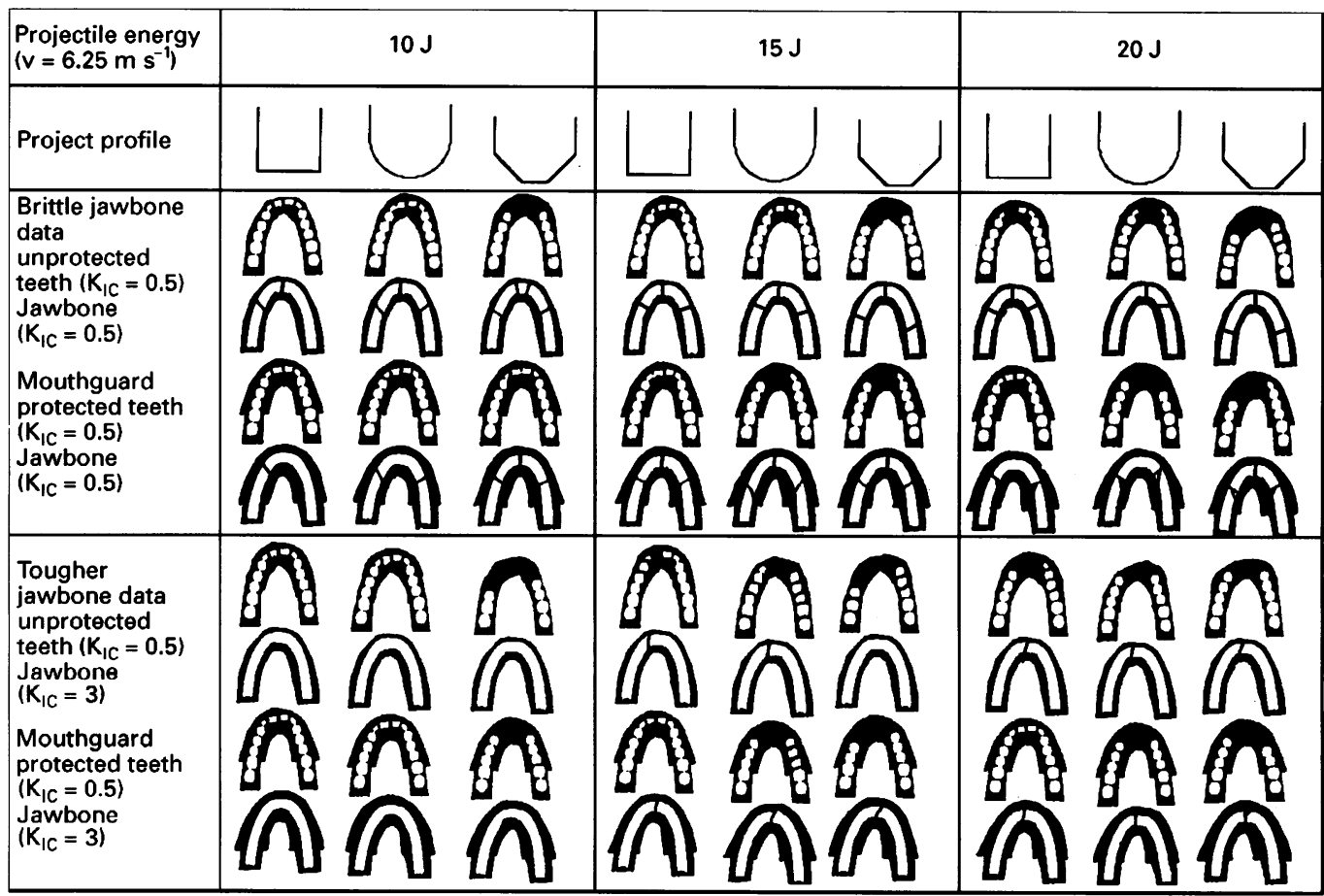

Figure 5 Impact test results (see text for description).

As can be seen in fig 5, increasing the kinetic energy of the projectile gives a general increase in the amount of damage in both the guarded and the unguarded condition. However, the rate of increase falls off once the projectile impact energy reaches $20 \mathrm{~J}$. It is considered that the test may become insensitive at this energy level and above, due to the extensive amount of damage imparted.

As expected, decreasing the nose radius of the projectile gave a more concentrated blow. This increases contact stresses for the same rate of decrease of projectile momentum and results in a significant increase in the impact damage. The effect of toughening up the jawbone was also seen to transfer damage to the teeth, indicating that deformation of the whole jaw is an insufficient energy absorption mechanism. This occurs in both the guarded and the unguarded condition and was particularly noticeable with the conical ended projectile contacting the guarded jaw with the lowest impact energy. With the brittle jaw, three fractures occurred in the jawbone and no teeth were fractured. Under similar impact conditions a tougher jaw survived intact but at a cost of four broken teeth.

It is considered that a conical ended projectile, with $10 \mathrm{~J}$ impact energy, impacting upon a standard jaw with the tougher jawbone offers the most promise in creating useful standard conditions. Such conditions reproduce clinical observation and give sufficient damage for comparative purposes. Under such conditions the jaw did not fracture in either the guarded or the unguarded condition. However, the number of broken teeth was reduced from an average of seven per blow to four per blow when the mouthguard was used. Hence any further improvements to mouthguard design would be expected to create a further level of damage reduction which would be reflected in a reduction in the numbers of fractured teeth observed. It is apparent that the ability of the guard to dissipate local deformation to a wider area of the standard jaw is important and in this respect the improved fit of custom made guards is claimed to be a major advantage. Presently such claims cannot be substantiated by quantitative and repeatable testing. It is intended to investigate the relative performance of "boil and bite" guards in the immediate future.

CONCLUSIONS

(1) A standard jaw, based on the design considered in this work and fitted with a custom made mouthguard offers some scope for future investigation of this complex impact situation.

(2) Careful selection of impact energy, projectile profile, jawbone fracture toughness, and tooth fracture toughness can give conditions that simulate clinical observation in a standardised jaw.

(3) At present, a conical ended, $10 \mathrm{~J}$ energy, $6.25 \mathrm{~m} \mathrm{~s}^{-1}$ velocity projectile, impacting a standard jaw where the simulated jawbone is considerably tougher than the tooth material, best reflects clinical observation and offers the best base from which a standard test could be developed.

This work has been supported from funding from the Department of Trade and Industry and we acknowledge the contributions made by Sheffield Orthodontic Laboratories and by Gordon Imlach and Ian Norman at the Open University Materials Department.

1 Scott J, Burke FJT, Watts DC. A review of dental injuries and the use of mouthguards in contact team sports. $B$ Dent F 1994;176:310-4.

2 DeYoung AK, Robinson E, Goodwin WC. Comparing comfort and wearability: custom-made vs self-adapted mouthguards. F Am Dent Assoc 1994;125:1112-8.

3 Lancaster DM, Ranelli DN. Comparative evaluation of colLancaster DM, Ranelli DN. Comparative evaluation of college football officials' attitudes toward NCAA regulation 
4 Chapman PJ, Nasser BP. Attitudes to mouthguards and prevalence of orofacial injuries in four teams competing at the second Rugby World Cup. Br F Sports Med 1993;27: the secon.

5 Croll TP. Custom-fitted protective mouthguards. $f$ Esthet Dent 1992;4:143-7.

6 Milward PJ, Jagger RG. A laminating procedure for thermoformed mouthguards. $\mathcal{F}$ Prosth Dent 1992:68:862-3.

7 Park JB, Shaull KL, Overton B, Donly KJ. Improving mouthguards. F Prosth Dent 1994;72:373-80.

8 De Wijn JR, Vrijhoef MMA, Versteegh PA, Stassen HP Linn EW. A mechanical investigation to the functioning of

\section{Commentary}

This article sets out to develop a standard test procedure for mouthguard assessment. A test rig is described, results taken, appropriate forces discussed, and a standardised method suggested. This should allow comparison between different types of mouth guard and improvements in design developed in the laboratory before being tested in the field.

R W KENDRICK mouthguards. Proceedings of 3rd General Meeting of the Europen Society of Biomechanics. In: Huiskes R, Van Campen D, De Wijn JR, eds. Biomechanics: principles and applications. The Hague: Martinus Nijhoff, 1982:451-58.

9 Matsuno T, Korshi M. Fracture toughness of porous sintered bodies of hydroxyapatite. Chem Lett 1992;12: 2335-8.

10 Takagi M, Mochida M, Uchida N, Saito K, Uematsu K. Filter cake forming and hot isostatic pressing for TZP199-203. dispersed hydroxyapatite composite. $\mathcal{F}$ Material Sci 1992;3

\section{Cor, what a team!}

At a recent interview, when asked to choose his greatest ever team performance, the former President of FIFA (Fictitious International Football Association) Sir Stanley Rous Sarcoma chose the 1997 Plymouth Argyll Robertson Pupil FC (a.k.a. "the Medics"). Their nickname arose from the clinical way they disposed of visitors to their home ground at Weber Parkes (Syndrome City). "The Medics" were no less successful away from home due to the surgical precision with which they dissected host defences on the ephemeral break. The club had already become financially extremely successful due to their Chairman Martin "Starr" Edwards-"Val(v)ue for money is the heart of the game" was his motto. The allimportant style of play, however, was dictated by the team's manager Graham Turner Syndrome (before his subsequent fall from grace due to involvement in the "XO" sex scandal). The team's crowning glory was winning the 1997 European Cup Final when a dramatic last minute penalty helped defeat the German side Bayer Munich at Lisbon's famous "Stadium of Light-Chained Paraprotein".

\section{The team (nicknames in brackets)}

1 Bob Wilson (Storage Disease) - initially an adolescent delinquent, he says he learnt the art of goalkeeping at an approved school (Lillishall) in order to avoid later excessive "Copper" problem.

2 Robert Jones (Procedure)-performances belied severe ankle instability problems.

3 Earl Barrett (Oesophagus) - often produced deep penetrating holes in defences.

4 Alan Hansen (Bacillus)-never seemed to feel pain when tackling opponents.

5 Tony Adams-Later of Arsenal, and formerly with Stoke, Adam's attacks were impressive but infrequent.

6 Jimmy Hill (Sachs Lesion)-the "hatchet-man" of the team.
7 Anders Limpar (Skin Disease - a great acquisition from the Swedish club Adipose Tuberosa Simplex FC.

8 Andy Grey-Turner's greatest signing.

9 Kevin Campbell (de Morgan) - always dependable from "the Spot".

10 Ian Wright-great goalscorer before his career was "Stained" by involvement in X-rated "blood films".

11 Robbie Fowler -Solution to most striking problems especially against the Arsen(ic)al.

\section{Subs}

12 Chris Bart-Williams (Haemoglobin)the perpetual baby of the side.

13 Paul Allen (Test) - great goal scorer but occasionally fades away when there are difficulties with adequate supply.

14 George Berry (Aneurysm)-once famed for his deft clips at corners now not so good in air owing to serious head injury.

15 Peter Osgood (Schlatter Disease)overcame knee injury early in career to later become a superstar.

16 Mark Hughes - reflex striking at its best but least said about private life! What a great team-assembled for less than the money it cost to buy one of the Newcastle United club feet! So famed was this team that at the end of their careers Wilson, Hansen, Hill, and Grey all became TV presenters on BBC's "Cross-Match of the Day". Ah, who will ever forget that night in Lisbon when Jones had to score that penalty "for the team" against the famed German International keeper Heinz (bodies)-Reidel (lobe) Bence. Jones showed nerves of Steel (-RichardsonOlsezewski) when slotting home that last minute spot-kick hence such a situation has gone down in football folklore as the Bence-Jones (Pro-team) Test.

W W GIBBON Football correspondent The Sun NHS (mis) Trust 